\title{
A!
}

This is an electronic reprint of the original article.

This reprint may differ from the original in pagination and typographic detail.

Li, Chi; Zhou, Xu; Zhai, Feng; Li, Zhenjun; Yao, Fengrui; Qiao, Ruixi; Chen, Ke; Yu, Dapeng;

Sun, Zhipei; Liu, Kaihui; Dai, Qing

Quiver-quenched optical-field-emission from carbon nanotubes

Published in:

Applied Physics Letters

DOI:

$10.1063 / 1.5003004$

Published: 25/09/2017

Document Version

Publisher's PDF, also known as Version of record

Please cite the original version:

Li, C., Zhou, X., Zhai, F., Li, Z., Yao, F., Qiao, R., Chen, K., Yu, D., Sun, Z., Liu, K., \& Dai, Q. (2017). Quiverquenched optical-field-emission from carbon nanotubes. Applied Physics Letters, 111(13), [133101].

https://doi.org/10.1063/1.5003004

This material is protected by copyright and other intellectual property rights, and duplication or sale of all or part of any of the repository collections is not permitted, except that material may be duplicated by you for your research use or educational purposes in electronic or print form. You must obtain permission for any other use. Electronic or print copies may not be offered, whether for sale or otherwise to anyone who is not an authorised user. 


\section{Quiver-quenched optical-field-emission from carbon nanotubes}

Chi Li, Xu Zhou, Feng Zhai, Zhenjun Li, Fengrui Yao, Ruixi Qiao, Ke Chen, Dapeng Yu, Zhipei Sun, Kaihui Liu, and Qing Dai

Citation: Appl. Phys. Lett. 111, 133101 (2017); doi: 10.1063/1.5003004

View online: https://doi.org/10.1063/1.5003004

View Table of Contents: http://aip.scitation.org/toc/apl/111/13

Published by the American Institute of Physics

\section{Articles you may be interested in}

Clocking plasmon nanofocusing by $\mathrm{THz}$ near-field streaking Applied Physics Letters 111, 131102 (2017); 10.1063/1.4991860

Single walled carbon nanotube-based stochastic resonance device with molecular self-noise source Applied Physics Letters 111, 133501 (2017); 10.1063/1.4986812

Single-shot non-intercepting profile monitor of plasma-accelerated electron beams with nanometric resolution Applied Physics Letters 111, 133105 (2017); 10.1063/1.4998932

Topology optimized gold nanostrips for enhanced near-infrared photon upconversion Applied Physics Letters 111, 133102 (2017); 10.1063/1.4998552

Effect of room temperature lattice vibration on the electron transport in graphene nanoribbons Applied Physics Letters 111, 133107 (2017); 10.1063/1.4999127

Edge effect enhanced photo-thermionic emission from a carbon nanotubes array Applied Physics Letters 110, 093105 (2017); 10.1063/1.4977189

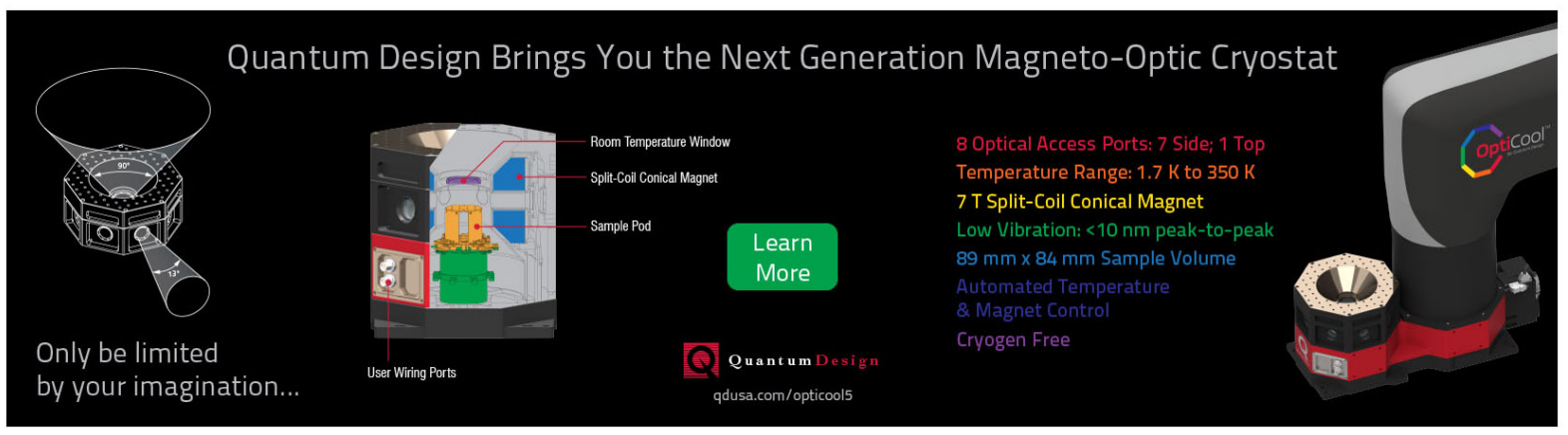




\title{
Quiver-quenched optical-field-emission from carbon nanotubes
}

\author{
Chi Li, ${ }^{1, a)}$ Xu Zhou, ${ }^{2, a)}$ Feng Zhai, ${ }^{3}$ Zhenjun Li, ${ }^{1}$ Fengrui Yao, ${ }^{2}$ Ruixi Qiao, ${ }^{2}$ Ke Chen, ${ }^{1}$ \\ Dapeng Yu, ${ }^{2}$ Zhipei Sun, ${ }^{4, b)}$ Kaihui Liu, ${ }^{2, b)}$ and Qing Dai ${ }^{1, b)}$ \\ ${ }^{1}$ National Center for Nano Science and Technology, Beijing 100190, China \\ ${ }^{2}$ School of Physics, Academy for Advanced Interdisciplinary Studies, Collaborative Innovation Center \\ of Quantum Matter, Peking University, Beijing 100871, China \\ ${ }^{3}$ Department of Physics, Zhejiang Normal University, Jinhua 321004, China \\ ${ }^{4}$ Department of Micro- and Nanosciences, Aalto University, Tietotie 3, FI-02150 Espoo, Finland
}

(Received 5 May 2017; accepted 1 September 2017; published online 25 September 2017; corrected 27 September 2017)

\begin{abstract}
Carbon nanotubes (CNTs) enable large electric field enhancement for an extremely broad bandwidth spanning from the optical domain down to static fields. This is due to their high aspect ratio, small tip radius, and high structural stability. CNTs therefore represent an ideal modelsystem for the investigation of nonlinear and strong-field phenomena. In this paper, we extend the range of optical-field-emission materials from metal nanostructures to CNTs. Quiver-quenched optical-field-emission (i.e., the transition to a sub-cycle regime) is observed for CNTs tips in a short-wavelength laser field of $820 \mathrm{~nm}$ that requires a mid-infrared excitation field of conventional metal tips emitters. This special property relies on the ultrasmall tips radius $(\sim 1 \mathrm{~nm})$ and the high optical-field enhancement ( 21.6) properties of CNTs. This study suggests that CNTs are excellent candidates for optically driven ultrafast electron sources with both high spatial and high temporal coherence. They also provide more freedom for the manipulation and control of electron dynamics at the attosecond timescale, which extends the bandwidth of light-wave electronic devices. Published by AIP Publishing. [http://dx.doi.org/10.1063/1.5003004]
\end{abstract}

Optical-field-emission from nanoscale solids are of fundamental interest for next-generation ultrafast electronmicroscopy and spectroscopy, ${ }^{1}$ compact coherent x-ray sources, ${ }^{2}$ and attosecond research, in general. ${ }^{3,4}$ The attraction of nanostructures relies on two closely connected features: the local geometrically mediated field enhancement and subwavelength confinement of optical-fields. ${ }^{5,6}$ Local field enhancement facilitates access to the optical-field-emission regime, ${ }^{7}$ whereas spatial localization has the potential to generate optical-field-driven electron dynamics exclusive to nanostructures. ${ }^{5,8,9}$ In the past decade, a few research groups have successfully accessed this regime employing nanoscale metal tips, and near-infrared, or mid-infrared pulses. ${ }^{10,11}$ More recently, increasing attention has been paid to certain features that originate in the sub-wavelength spatial confinement of optical-fields. ${ }^{5,12}$ Specially, a sub-cycle electron dynamic regime has been discovered for the mid-infrared field $(>2 \mu \mathrm{m})$, in which the field localization scale approaches the electron's quiver amplitude. ${ }^{5}$ Such conditions enable interesting optically driven electron-dynamics, which involve quiver-quenched optical-field-emission ${ }^{5}$ and directional emission. ${ }^{13}$ However, research into this regime in the short-wavelength field (visible to near-infrared) is still being conducted because it is important for the attosecond precision control of electron beams. ${ }^{6,14}$

The underlying principles of optical-field-driven have been studied widely. They consist of two individual steps, the liberation of electrons from a solid, and the subsequent electron propagation and acceleration within the incident

\footnotetext{
${ }^{a)} \mathrm{C}$. Li and X. Zhou contributed equally to this work.

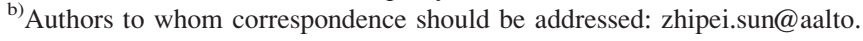
fi;khliu@pku.edu.cn; and daiq@nanoctr.cn.
}

strong optical-field. ${ }^{15}$ The first step is governed by the Keldysh adiabaticity parameter ${ }^{16} \gamma=\omega / \omega_{t}$, where $\omega_{t}=e F /$ $\sqrt{2 m \Phi}$ characterizes the photon-driven (quantum, $\gamma>1$ ) and field-driven (classical, $\gamma<1$ ) regimes; $\omega$ is the circular optical frequency, and $F$ is the localized field enhanced from the optical-field $F_{0}$ due to the enhancement factor $\beta$, which is achieved through the engineered tip-sharpness with a radius of curvature, $R$. The second step is characterized by another dimensionless adiabaticity parameter ${ }^{5}\left(\delta=l_{F} / l_{q}\right)$ that relates the $(1 / e)$-decay length $l F$ of the optical-field, which scales with the emitting tip-radius $R$, and the electron quiver amplitude $l q=e F / m \omega^{2}$. In an optical cycle, three different electron dynamics are expected to occur, depending on the emitting phase [see Figs. 1(a) and 1(b)]. For $\delta>1$, the bulk of the electron population quiver in a nearly homogeneous field occurs during multiple optical cycles in the usual way, while the electrons occasionally scatter off the emitting surface. For $\delta<1$, a large proportion of the emitted electrons rapidly escape from the tip-enhanced field within a timeframe much shorter than an optical cycle. It is clear that
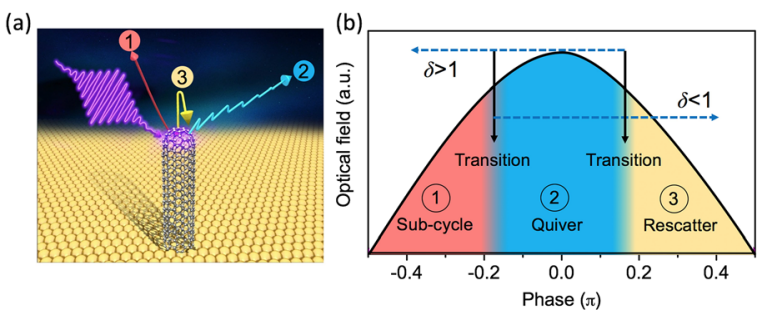

FIG. 1. (a) Schematic diagram of optical-field-driven from a CNT tip, including three possible electron-trajectories, 1: sub-cycle, 2: quiver, and 3: rescatter. (b) Phase-dependent electron trajectories, 1: sub-cycle in earlier phase, 2: quiver in middle phase, and 3: rescatter in later phase, the transitions between two adjacent dynamics moves with $\delta$. 
$\beta, R, \omega$, and $F_{0}$ are the four key-parameters that determine the mechanism of both steps, critically strengthening the dominant emission process. To reach into the strong-field regime and subcycle regime, higher $\beta, F_{0}$, and lower $R, \omega$ values are required.

High $\beta$ and low $R$ are readily facilitated using carbon nanotubes (CNTs) as the electron source. The CNT tip radius-of-curvature can easily be synthetized down to subnanometers. At this scale, the electrical conductivity can be orders of magnitude higher than its equivalently sized metallic counterpart-even for the most conductive of metals. ${ }^{17}$ In addition, the excellent thermal and mechanical properties are retained, which ensure the emitter's robustness when exposed to the aggressive emission-conditions and associated high fields. ${ }^{18}$ These factors make carbon nanotubes a very promising light-matter interaction material. Benefitting from these unique properties, in our previous work, the optical-field-emission has been accessed from CNTs (with a radius of $\sim 1 \mathrm{~nm}$ ) at $410 \mathrm{~nm}$ laser with a narrow electron energy spread of $0.25 \mathrm{eV}$. In this paper, we attempt to access the quiver-quenched optical-field-emission (i.e., sub-cycle regime) at $820 \mathrm{~nm}$ laser, which is much shorter that required for conventional metal tips. ${ }^{5}$

Vertically aligned CNT cluster arrays [Fig. 2(a)] were grown on a highly doped n-type silicon chip using chemical vapor deposition (CVD). ${ }^{19}$ The silicon substrate was first coated with an $\mathrm{Al}(10 \mathrm{~nm}) / \mathrm{Fe}(1 \mathrm{~nm})$ multilayer catalyst deposited via sputtering. The substrate was then heated to $900^{\circ} \mathrm{C}$, at $10^{-2}$ mbar. Acetylene was chosen as the carbon feedstock and introduced to the deposition chamber after the temperature had reached $900^{\circ} \mathrm{C}$. The growth process lasted for $1 \mathrm{~min}$. After the growth process, the samples were annealed in hydrogen at $1000^{\circ} \mathrm{C}$ for $2 \mathrm{~h}$ to remove amorphous carbon deposits and other impurities.

The morphology and structure of the CNT sample was analyzed using a scanning electron microscope (SEM, Hitachi S-4800) and a high-resolution transmission electron (a)
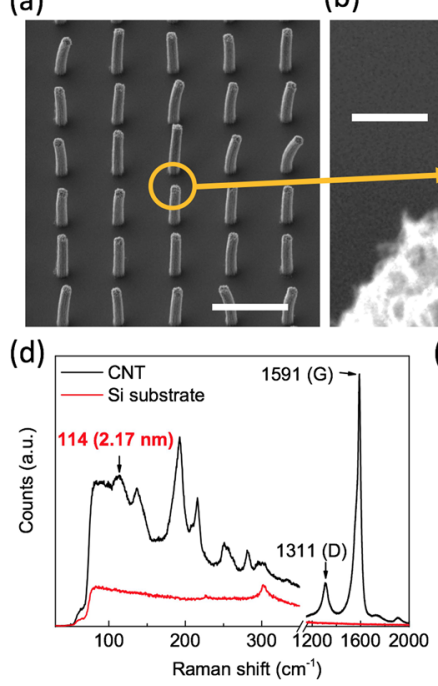

(b)

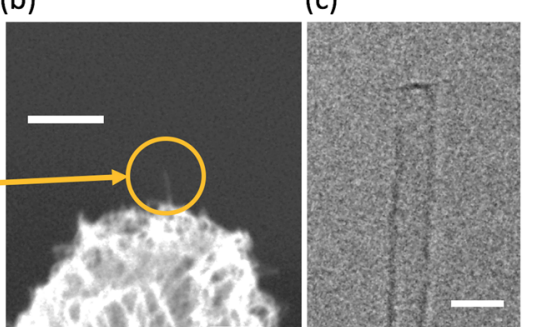

(e)

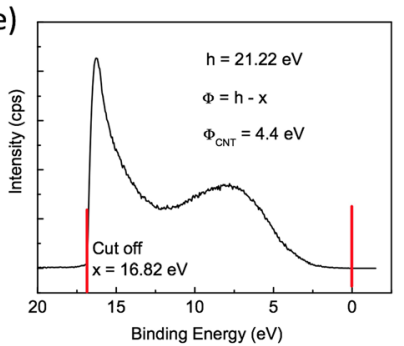

FIG. 2. (a) SEM image of a large area CNTs array. The scale-bar is $10 \mu \mathrm{m}$. (b) A high-magnification SEM image of the apex. The scale bar is $1 \mu \mathrm{m}$. (c) HRTEM image of a typical CNT. The scale bar is $3 \mathrm{~nm}$. (d) Raman spectrum of a typical as-grown CNT sample. (e) Work function measurement of asgrown CNTs. microscope (HRTEM, FEI Tecnai F20). The diameters of the CNTs were determined based on their radial breathing mode frequency $\left[\omega_{\mathrm{RBM}}=248 / \mathrm{d}\left(\mathrm{cm}^{-1} / \mathrm{nm}\right)\right]$ using Raman spectroscopy. ${ }^{20}$ The pristine CNTs were dispersed in absolute alcohol via ultrasonication and then drop transferred onto $300 \mathrm{~nm} \mathrm{SiO}_{2} / \mathrm{Si}$ substrates. Raman spectra were acquired using $\mathrm{He}-\mathrm{Ne}$ laser $(632.8 \mathrm{~nm})$ excitation, and the data were recorded using a confocal micro-Raman spectrometer (HORIBA Jobin Yvon, LabRam HR 800). Ultraviolet Photoelectron Spectroscopy (UPS, AXIS ULTRA DLD) was used to determine work functions. A He discharge-source $(21.22 \mathrm{eV})$ was used with a resolution of $\sim 0.2 \mathrm{eV}$.

The experimental setup is shown in Fig. S3. The laser light $(820 \mathrm{~nm}, 100 \mathrm{fs}$, and $80 \mathrm{MHz})$ hits the CNT tip from the side at a nearly $90^{\circ}$ angle. It was focused to a $2.50 \mu \mathrm{m}$ (FWHM) spot at one CNT cluster apex. The laser beam was focused by scanning the emission current. The light was linearly polarized, and the polarization angle was controlled via a half-wave plate. The electron emission experiments have been done in a high-vacuum chamber $\left(10^{-7}\right.$ Torr). The anode was placed $400 \mu \mathrm{m}$ away from the cathode using a thick mica insulating spacer. A Keithley 2400 source-measurement unit (SMU) was used to bias the anode with voltages up to $200 \mathrm{~V}$ and measure the anode current. The current-measurements presented in the main text are those recorded at the anode.

The growth process resulted in $10 \mu \mathrm{m}$ tall CNT clusters of defined areal patterns-see Fig. 2(a). Although the as-grown clusters contain many nanotubes, the growth kinetic was such that a few individual tubes protruded (repeatedly between growths) from these clusters to produce a few isolated nanoscopic apexes [see Fig. 2(b)], which are believed to be the main emission sites to facilitate the extremely high fieldenhancement there. The CNTs have a tip radius of $\sim 1 \mathrm{~nm}$, as shown in transmission electron images [see Fig. 2(c)] and confirmed by their Raman spectrum [see Fig. 2(d)]. The radius of the CNT was considered to be $1 \mathrm{~nm}$. The work function of the CNTs was measured to be $\sim 4.4 \mathrm{eV}$ [see Fig. 2(e)].

To describe the electron dynamics, the field-enhancement factor is required to calculate the enhanced local field. Therefore, the emission current as a function of laser power (I-P curves) has been measured and recorded using a doublelogarithmic scale [see Fig. 3(a)]. For a low emission current, a nonlinear laser-power (and corresponding intensity) dependence is observed, which approximately follows the fifthorder power law (dashed line) of a multiphoton process. ${ }^{14,21}$ However, the measured work function $(\Phi)$ of the CNT (a)

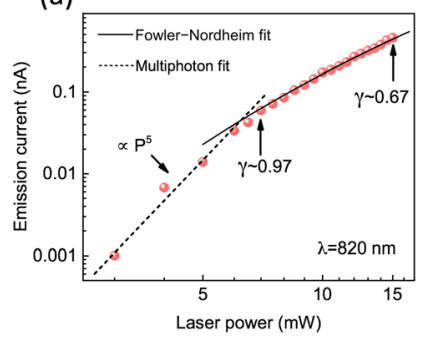

(b)

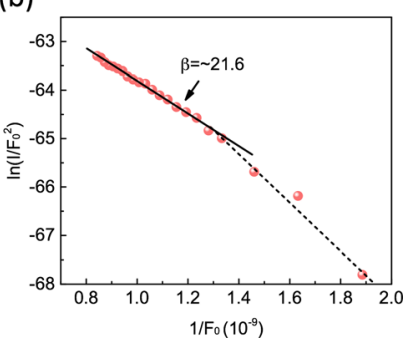

FIG. 3. (a) Emission current as a function of laser power, biased with $50 \mathrm{~V}$. For low-power range, a fifth power is observed, while a lower-power function is obtained for the higher power range. (b) Fowler-Nordheim (FN) fitting of the optically driven emission-current, with a field enhancement factor $(\beta)$ of $\sim 21.6$. 
$(\sim 4.4 \mathrm{eV})$ requires only three photons $(4.8 \mathrm{eV})$ for photoemission. The enhanced emission-nonlinearity indicates that electrons originate almost exclusively from the sharp CNT tips. ${ }^{7}$ Around $7 \mathrm{~mW}$ (and an incident peak intensity of about $18 \mathrm{GW} / \mathrm{cm}^{2}$, and a laser field of about $0.81 \mathrm{GV} / \mathrm{m}$ ), the curve deviates from this behavior and changes to a lower power law dependence. This represents a transition from multiphoton photoemission to optical-field-emission. In the quasi-static optical-field-emission regime, the emission current approximately follows the static field-emission rate, which is given by cycle-averaged Fowler-Nordheim equation [fitting shown in Fig. 3(a)]: ${ }^{22}$

$$
I=\frac{A\left(\beta F_{0}\right)^{2}}{\Phi} \exp \left(\frac{-B \Phi^{\frac{3}{2}}}{\beta F_{0}}\right)
$$

where $I$ is the emission current, $A=1.56 \times 10^{-6} \mathrm{~A} \mathrm{~V}^{-2} \mathrm{eV}$, $B=6.83 \times 109 \mathrm{~V} \mathrm{eV}^{-3 / 2} \mathrm{~V} \mathrm{~m}^{-1}, \beta$ is a field enhancement factor, ${ }^{23} \Phi$ is the work function, and $F_{0}$ is incident laser field. The FN plot derived from the above current-field curve demonstrates a high degree of linearity, as shown in Fig. 2(c), which further confirms that the dominant emission has fielddriven behavior. Thus, the field enhancement factor of bare CNTs $(\beta)$ can be calculated from the slope $(\mathrm{S})$ of the linearized FN data, using the transformed form of Eq. (1)

$$
\ln \left(\frac{I}{F_{0}^{2}}\right)=\ln \left(\frac{A \beta^{2}}{\phi}\right)-\left(\frac{B \phi^{\frac{3}{2}}}{\beta}\right)\left(\frac{1}{F_{0}}\right),
$$

and thus

$$
\mathrm{S}=\frac{-\mathrm{B} \Phi^{\frac{3}{2}}}{\beta}
$$

The calculated $\beta$ was $\sim 21.6$. Accordingly, the calculated $\gamma$ at $7 \mathrm{~mW}$ is $\sim 0.96$. This confirms our conclusion of a fielddriven process. Thus, both the cycle-averaged FN-fitting and calculated Keldysh $\gamma$, fully confirm that the emission is operating in the optical-field-driven tunneling regime at 7-15 $\mathrm{mW}$ for $820 \mathrm{~nm}$ excitation.

As mentioned earlier, the optical-field strength constitutes one of the control parameters for the transition from quiver to sub-cycle electron-dynamics. The $\delta$-parameter scales proportionally to $\mathrm{F}^{-1}$ at fixed wavelengths. However, because the experimentally accessible intensity-range is limited by damage thresholds, access to the sub-cycle regime by increasing $\mathrm{F}$ is expected to be difficult, especially for a shortwavelength excitation field. Fortunately, CNT emitters studied in this work have a much higher $\beta$ and a much smaller $R$ compared to conventional metal tips, which facilitate access into a sub-cycle regime. As shown in Fig. 4(a) (up panel), the calculated $\delta$ decreased from 0.78 to 0.53 with the laser power increasing from $7 \mathrm{~mW}$ to $15 \mathrm{~mW}$. This indicates that optical-field-emission has clearly entered the sub-cycle regime for the present measurement range.

To further strengthen our speculation, the electron energy spectra at different laser-intensities were measured using the retarding field method. ${ }^{5}$ As depicted in Fig. 4(b) (dotted line), the cutoff kinetic energies for the present (a)
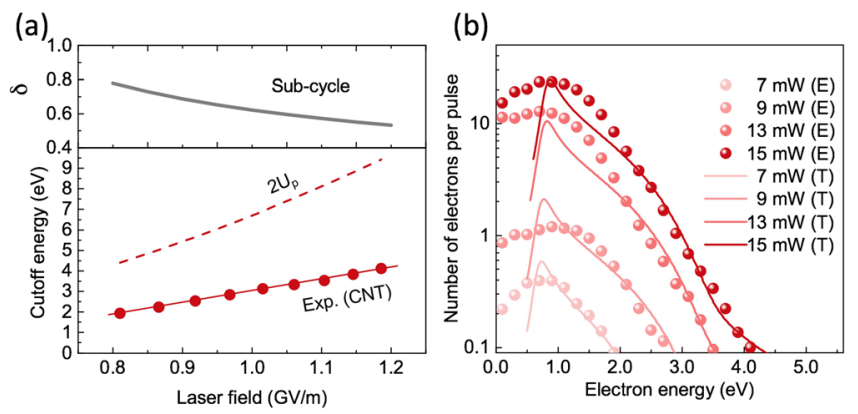

FIG. 4. (a) Spatial adiabaticity parameter $\delta$ as a function of the incident laser field used in the experiments (upper panel). Cutoff kinetic energies versus incident laser field (bottom panel) for emission from CNT in the experiments (dots) shows a linear behavior and deviation from that of a homogeneous field ( $2 \mathrm{U}_{\mathrm{p}}$, dashed line). (b) Experimental (dots, E) and simulated (solid lines, T) kinetic energy distributions of emitted electrons from CNTs for increasing power and a wavelength of $820 \mathrm{~nm}$.

CNT-based ultrafast electron source increases with laser intensity. To examine this behavior further, we computed the kinetic-energy spectrum using experimentally derived parameters in a two-step model adapted to localized optical-fields (see supplementary materials for details). The simulation includes a simplified FN tunneling model (first step-electron tunneling) and the interaction of the electrons with a strongly localized field near the tip (second step-electron propagation). The results are shown in Fig. 4(b) (solid line). In this study, the high energy electrons induced through rescattering ${ }^{5}$ were not considered in the simulation because the backscattering efficiency of electrons on the carbon surface was very low. ${ }^{24}$ Good agreement with the experimental data was obtained, which strongly supports our sub-cycle conclusion. In the subcycle regime, the cutoff energies, as recorded in Fig. 4(a) (bottom panel), display a linear dependency on the laser field and greatly deviate from the $2 \mathrm{U}_{\mathrm{p}}$ (ponderomotive potential, $\mathrm{U}_{\mathrm{p}}=e^{2} F^{2} / 4 m \omega^{2}$ ) for a homogeneous field and the same driving field. This strongly suggests that the electron emission originates from a strongly localized field. This conclusion is also consistent with previous findings reported for mid-infrared field. ${ }^{25}$ In addition, we simulated the electron dynamics with a fixed local (enhanced) field, tip radius, and wavelength. We found that, for the present electron emission system, most trajectories display sub-cycle features (red lines) and minimal quiver motion (blue lines). In addition, back acceleration (yellow lines) occurs throughout a reduced range of emission phases [see Fig. 5(a)]. For comparison, we also simulated the electron

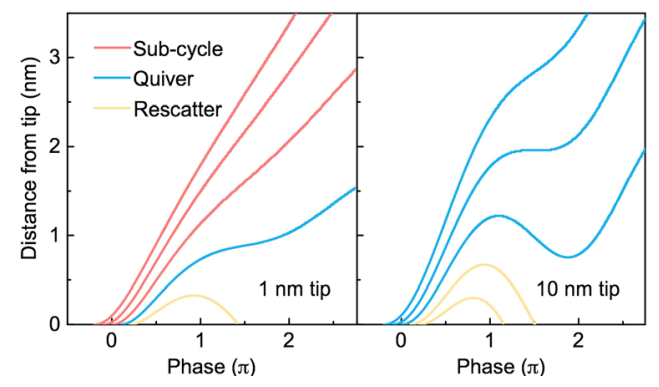

FIG. 5. Simulated electron trajectories for five emission phases $(-0.2 \pi$, $-0.1 \pi, 0 \pi, 0.1 \pi, 0.2 \pi)$, from a $1 \mathrm{~nm}$ tip and $10 \mathrm{~nm}$ tip. 
dynamics with a tip radius of $10 \mathrm{~nm}$ for the same local field and wavelength [see Fig. 5(b)]. This produces much stronger quiver-motions.

In conclusion, the sub-cycle regime for optical-fieldemission is successfully accessed with a much shorter excitation-field wavelength of $820 \mathrm{~nm}$ from CNT tips with $1 \mathrm{~nm}$ radius. This required mid-infrared pulses in previously reported studies and has been shown to benefit from the high field enhancement factor and sub-nano near-field decaylength. For the present conditions, the sub-cycle regime can be accessed with shorter visible-light excitation. It is hoped that further studies (at extreme conditions) of single CNT emitters and plasmonically active samples will further clarify the underlying electron emission processes. Our findings make it possible to use high spatially coherent electronsources with high temporal-resolution for time-resolved electron-microscopy and diffraction, and free-electron lasers. They may also open doors for "light-wave electronics" at extended bandwidths.

See supplementary material for complete simulation.

This work was supported by National Key R\&D Program of China (Grant Nos. 2016YFA0202000, 2015CB932400, and 2016YFA0300903), the National Natural Science Foundation of China (Grant Nos. 11427808, 51372045, 51602071, 11474006, 5152220, and 91433102), Beijing science and technology projects (Grant No. Z161100002116016), the International Science and Technology Cooperation Project (No. 2014DFR10780, China), the Academy of Finland (Grant Nos.: 276376, 284548, 295777, and 304666), TEKES (OPEC), Nokia foundation, the Oppenheimer Trust, and the European Union's Seventh Framework Programme (Grant No.: 631610).

${ }^{1}$ A. H. Zewail, Science 328, 187 (2010).

${ }^{2}$ H. P. Freund, D. C. Nguyen, and B. Carlsten, Phys. Rev. Spec. Top.Accel. Beams 15, 030704 (2012).
${ }^{3}$ V. Gruson, L. Barreau, A. Jimenez-Galan, F. Risoud, J. Caillat, A. Maquet, B. Carré, F. Lepetit, J.-F. Hergott, T. Ruchon et al., Science 354, 734 (2016).

${ }^{4}$ F. Krausz and M. Ivanov, Rev. Mod. Phys. 81, 163 (2009).

${ }^{5}$ G. Herink, D. R. Solli, M. Gulde, and C. Ropers, Nature 483, 190 (2012).

${ }^{6}$ M. Kruger, M. Schenk, and P. Hommelhoff, Nature 475, 78 (2011).

${ }^{7}$ R. Bormann, M. Gulde, A. Weismann, S. V. Yalunin, and C. Ropers, Phys. Rev. Lett. 105, 147601 (2010).

${ }^{8}$ A. L. Cavalieri, N. Muller, T. Uphues, V. S. Yakovlev, A. Baltuska, B. Horvath, B. Schmidt, L. Blumel, R. Holzwarth, S. Hendel et al., Nature 449, 1029 (2007).

${ }^{9}$ M. Schultze, E. M. Bothschafter, A. Sommer, S. Holzner, W. Schweinberger, M. Fiess, M. Hofstetter, R. Kienberger, V. Apalkov, V. S. Yakovlev et al., Nature 493, 75 (2013).

${ }^{10}$ M. Schenk, M. Kruger, and P. Hommelhoff, Phys. Rev. Lett. 105, 257601 (2010).

${ }^{11}$ P. Dombi, A. Horl, P. Racz, I. Marton, A. Trugler, J. R. Krenn, and U. Hohenester, Nano Lett. 13, 674 (2013).

${ }^{12}$ B. Piglosiewicz, S. Schmidt, D. J. Park, J. Vogelsang, P. Gross, C. Manzoni, P. Farinello, G. Cerullo, and C. Lienau, Nat. Photonics 8, 38 (2014).

${ }^{13}$ D. J. Park, B. Piglosiewicz, S. Schmidt, H. Kollmann, M. Mascheck, and C. Lienau, Phys. Rev. Lett. 109, 244803 (2012).

${ }^{14}$ M. E. Swanwick, P. D. Keathley, A. Fallahi, P. R. Krogen, G. Laurent, J. Moses, F. X. Kärtner, and L. F. Velásquez-García, Nano Lett. 14, 5035 (2014).

${ }^{15}$ P. B. Corkum, Phys. Rev. Lett. 71, 1994 (1993).

${ }^{16}$ L. V. Keldysh, Sov. Phys. JETP 21, 1135 (1965).

${ }^{17}$ A. Javey, J. Guo, Q. Wang, M. Lundstrom, and H. J. Dai, Nature 424, 654 (2003).

${ }^{18}$ R. Saito, G. Dresselhaus, and M. S. Dresselhaus, Physical Properties of Carbon Nanotubes (Imperial College Press, London, 1998).

${ }^{19}$ Z. J. Li, X. X. Yang, F. He, B. Bai, H. Zhou, C. Li, and Q. Dai, Carbon 89, 1 (2015).

${ }^{20}$ K. H. Liu, X. P. Hong, M. H. Wu, F. J. Xiao, W. L. Wang, X. D. Bai, J. W. Ager, S. Aloni, A. Zettl, E. G. Wang et al., Nat. Commun. 4, 1375 (2013).

${ }^{21}$ R. G. Hobbs, Y. Yang, A. Fallahi, P. D. Keathley, E. De Leo, F. X. Kartner, W. S. Graves, and K. K. Berggren, ACS Nano 8, 11474 (2014).

${ }^{22}$ R. G. Hobbs, Y. Yang, P. D. Keathley, M. E. Swanwick, L. F. VelasquezGarcia, F. X. Kartner, W. S. Graves, and K. K. Berggren, Nanotechnology 25, 465304 (2014).

${ }^{23}$ F. Kusa, K. E. Echternkamp, G. Herink, C. Ropers, and S. Ashihara, AIP Adv. 5, 077138 (2015).

${ }^{24}$ L. Huang, S. P. Lau, H. Y. Yang, and S. F. Yu, Nanotechnology 17, 1564 (2006).

${ }^{25}$ K. E. Echternkamp, G. Herink, S. V. Yalunin, K. Rademann, S. Schafer, and C. Ropers, Appl. Phys. B 122, 80 (2016). 